\title{
Polypoid Melanoma: Towards a Dermoscopic Approach
}

\author{
Camilo Rojas-Erazo ${ }^{1}$, Fernando Valenzuela², Laura Carreño ${ }^{3}$, Francisco González-Coloma²
}

1 Puerto Montt Hospital, Reloncavi Health Service, Puerto Montt, Chile

2 Department of Dermatology, Faculty of Medicine, University of Chile, Santiago, Chile

3 Pathology service, Clinical Hospital of the University of Chile, Santiago, Chile

Citation: Rojas-Erazo C, Valenzuela F, Carreño L, González-Coloma F. Polypoid melanoma: towards a dermoscopic approach. Dermatol Pract Concept. 2021:11(4); e2021112. DOI: https://doi.org/10.5826/dpc.1104a112

Accepted: March 8, 2021; Published: October 2021

Copyright: (92021 Rojas-Erazo et al. This is an open-access article distributed under the terms of the Creative Commons Attribution License BY-NC-4.0, which permits unrestricted noncommercial use, distribution, and reproduction in any medium, provided the original authors and source are credited.

Funding: None.

Competing interests: None.

Authorship: All authors have contributed significantly to this publication.

Corresponding author: Francisco González-Coloma, Department of Dermatology, Faculty of Medicine, University of Chile, Santiago, Chile. Email: fgonzalezcoloma@gmail.com

\section{Case Presentation}

A 75 -year-old man presented with a 2-inch exophytic lesion on the right gluteus with 1 year of progressive growth (Figure 1, A and B). Dermoscopy showed an irregular serohematic crusty surface that impaired the observation of atypical polymorphic vessels and a peduncle with multicolored pattern: diffuse red and white background with irregular yellow, brown, and black areas (Figure 1C). Erythematous papules of the ipsilateral coxal region had a central reddish homogeneous pattern with a peripheral pigmented rim at dermoscopy (Figure 1D). Histopathology revealed a polypoid melanoma, Clark V level, Breslow thickness of
$14 \mathrm{~mm}, 12$ mitoses $/ \mathrm{mm}^{2}$, extensive ulceration, and perineural invasion. Coxal papules were satellite metastasis. The dissemination study was negative, yet limited, because of the patient's death, 1 month later.

\section{Teaching Point}

This rare and aggressive variant of nodular melanoma can be dermoscopically distinguished from clinically similar tumors by the recognition of irregular crusted-fibrinous surfaces, atypical polymorphic vessels, blue-white veils in the exophytic portion, a multicolored pattern, and blue-gray nests at the base $[1,2]$. 


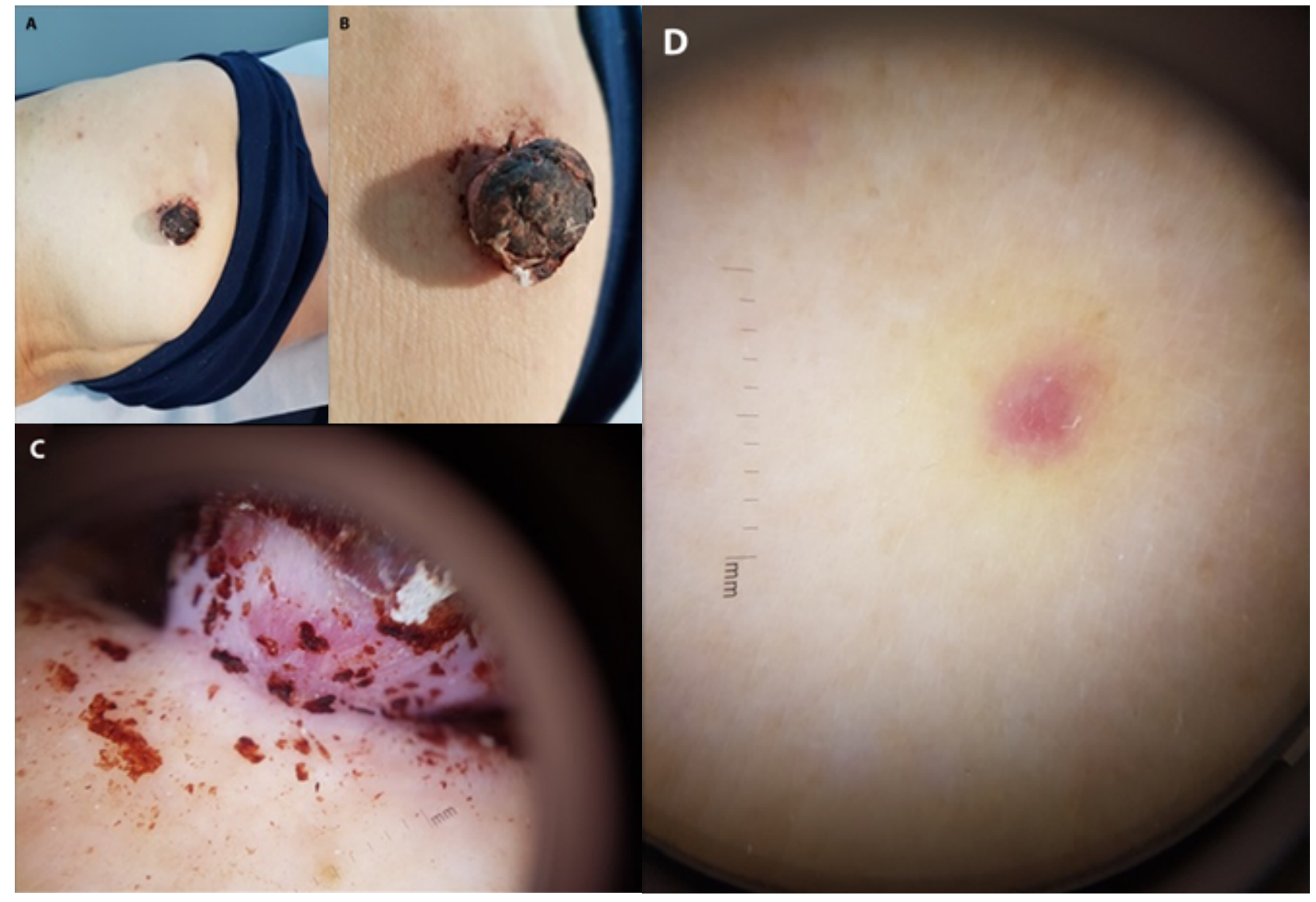

Figure 1. (A) Tumoral lesion on the right gluteus and erythematous papules on the ipsilateral coxal region. (B) Crusty and pigmented surface of the tumor. (C) Polarized dermoscopy (DermLite DL4W, magnification x10) reveals a multicolored pattern in the peduncle of the tumor and (D) homogeneous reddish center with pigmented periphery of satellite metastasis.

\section{References}

1. Cabrera R, Recule F. Unusual Clinical Presentations of Malignant Melanoma: A Review of Clinical and Histologic Features with Special Emphasis on Dermatoscopic Findings. Am J Clin Dermatol. 2018;19(s1):15-23. DOI:10.1007/s40257-018-0373-6. PMID: 30374898.
2. Di Altobrando A, Patrizi A, Dika E, Savoia F. Cauliflower-like exophytic mass on the skin: polypoid melanoma. Clinical, dermoscopic, and histologic features. An Bras Dermatol. 2020;95(6):748750. DOI:10.1016/j.abd.2020.04.010. PMID: 33041156. 